\title{
The frequency and prognostic effect of TERT promoter mutation in diffuse gliomas
}

\author{
Yujin Lee ${ }^{1}$, Jaemoon Koh², Seong-Ik Kim', Jae Kyung Won', Chul-Kee Park ${ }^{3}$, Seung Hong Choi ${ }^{4}$ \\ and Sung-Hye Park ${ }^{1,2,5^{*}}$ (D)
}

\begin{abstract}
Mutations in the telomerase reverse transcriptase gene promoter (TERTp) are common in glioblastomas (GBMs) and oligodendrogliomas (ODGs), and therefore, have a key role in tumorigenesis and may be of prognostic value. However, the extent of their prognostic importance in various gliomas is controversial. We studied 168 patients separated into five groups: Group 1: 65 patients with ODG carrying an IDH1 or IDH2 mutation (IDH-mutant) and 1p/19q-codeletion, Group 2: 23 patients with anaplastic astrocytoma (AA), IDH-mutant, Group 3: 13 patients with GBM, IDH-mutant, Group 4: 15 patients with AA, IDH-wildtype (WT), and Group 5: 52 patients with GBM, IDH-WT. TERTp mutations were found in 96.9\%, 4.4\%, 76. $9 \%, 20.0 \%$, and $84.6 \%$ of patients in Groups 1, 2, 3, 4, and 5, respectively. The R132H mutation in IDH1 was found in 60.5\% (23/38) of patients in the AA cohort (Groups 2 and 4) and 20.0\% (13/65) of patients from our GBM cohort (Groups 3 and 5), whereas all patients with ODG (Group 1) had a mutation either in IDH1 $(n=62)$ or IDH2 $(n=3)$. Using Kaplan Meier survival analysis, we found that the TERTp mutation was correlated with poor overall survival (OS) in Groups 2 and 4 combined $(P=0.001)$ and in Group $4(P=0.113)$, and in multivariate analysis, the TERTp mutant group was associated with significantly poor survival in Group $5(P=0.045)$. However, IDH mutation, MGMT methylation, and younger patient age ( $<55$ years old) were significantly correlated with favorable OS (all $P<0.05)$ in our cohort of astrocytic and ODGs. In patients with ODG (Group 1), mutant IDH and TERTp did not have prognostic value because these mutations were universally present. Based on the revised 2016 WHO classification of gliomas, we found that TERTp mutation was frequently present in patients with GBM or ODG and because it was strongly correlated with poor survival outcome in patients with IDH-WT GBM in multivariate analysis, it may be of prognostic value in this subgroup of patients with gliomas.
\end{abstract}

Keywords: Telomerase reverse transcriptase, Alpha-thalassemia/mental retardation syndrome X-linked (ATRX) , Anaplastic astrocytoma, Glioblastoma, Isocitrate dehydrogenase (IDH), Oligodendroglioma

\section{Introduction}

Gliomas are the most common primary malignant tumor of the central nervous system (CNS), and are comprised of diffuse astrocytic and oligodendroglial tumors (ODG). Significant research in the pathogenesis of gliomas revealed that various morphological phenotypes are related to underlying molecular genetic variations $[4,26]$. This understanding has resulted in the restructuring of glioma classification with the incorporation of genetically defined entities and histology [19].

\footnotetext{
* Correspondence: shparknp@snu.ac.kr

'Department of Pathology, Seoul National University Hospital, 103 Daehak-ro, Jongno-gu, Seoul 110-799, Republic of Korea

${ }^{2}$ Department of Pathology, Seoul National University College of Medicine, Seoul, Republic of Korea

Full list of author information is available at the end of the article
}

Of the many molecular changes found in gliomas, mutations in $I D H 1$ or IDH2 occur early during glioma formation, and are associated with better overall survival (OS). In addition, glioma subtypes can be identified by the IDH1/IDH2 mutation [11, 27]. The second most important genetic alteration is a co-deletion at chromosome regions $1 \mathrm{p}$ and $19 \mathrm{q}$, which results from a chromosomal translocation $\mathrm{t}(1 \mathrm{p} ; 19 \mathrm{q})(\mathrm{q} 10 ; \mathrm{p} 10)$, and is exclusively associated with ODGs and better prognosis [20]. Such examples demonstrate that the identification of new molecular genetic changes, which occur during glioma formation or development, are necessary to better guide clinical decision making.

Recently, mutations in the promoter region of the telomerase reverse transcriptase (TERTp) gene have been found in various cancers, including glioblastomas 
(GBMs) [18] and ODGs [3, 8]. Telomerase activity has a major role in tumorigenesis [6] and contributes to tumor development in different brain tumors, including astrocytomas, GBMs, and ODGs [12, 17]. The two most common mutations in the TERTp are C228T and C250T, which are located -124 base pairs (bp) and $-146 \mathrm{bp}$ respectively, upstream of the TERT ATG start site (chr5p15.33: 1,295,228 $\mathrm{C}>\mathrm{T}$ and 1,295,250 $\mathrm{C}>\mathrm{T}$, respectively) [15]. These mutations confer unrestricted growth properties to tumor cells by elongation of telomere length because of TERT activation, which indicates the importance of its role in the anti-senescence and immortal cancer development $[2,7]$.

It has been reported that the effects of TERTp mutations are inversely correlated with mutations found in the IDH1 gene, [22] which is a well-known molecular factor for a favorable prognosis [27]. Therefore, several studies have independently proposed that mutations in TERTp are associated with poor prognosis [15]. However, recently Pekmezci et al. studied TERTp and ATRX mutation status and their prognostic value in large cohorts from the UCSF and Mayo clinics and The Cancer Genome Atlas (TCGA), and found that the wildtype (WT) TERTp group was associated with good prognosis only in IDH1/IDH2 WT (IDH-WT) astrocytomas (grade II and III), while in other groups such as $I D H$-mutant astrocytomas (grades II and III) and GBMs (grade IV) with or without $I D H$-mutation, TERTp mutation status was not a statistically significant prognostic factor [23]. In addition, they found that ATRX was a good prognostic factor only in IDH-WT GBMs (grade IV), but not in any other glioma group [23].

In this study, we examined TERTp mutation status in a cohort of patients with either diffuse astrocytomas (grade III or IV) or ODG (grade II or III) to identify the frequencies of these mutations in our cohort and to evaluate their potential prognostic value, risk stratification, and future target therapy.

\section{Materials and methods}

Our cohort consisted of 168 patients in five groups, including 65 patients with ODGs carrying an $I D H$-mutation (IDH-mutant) and $1 \mathrm{p} / 19 \mathrm{q}$-codeletion (termed Group 1 or the ODG group), 23 patients with anaplastic astrocytoma (AA), $I D H$-mutation (Group 2), 13 patients with GBM, $I D H$-mutant (Group 3), 15 patients with AA, $I D H$-WT (Group 4), and 52 patients with GBM, IDHWT (Group 5), which were obtained from the archives of the Department of Pathology of the Seoul National University Hospital (SNUH) from 2007 to 2015. All patients underwent surgery at the Department of Neurosurgery at the SNUH. All tumors were reviewed by two neuropathologists (SH Park and JK Won) according to the revised 2016 World Health Organization (WHO) classification of tumours of the CNS [19]. The mean age of patients with ODG, IDH-mutant AA, IDH-mutant GBM, $I D H$-WT AA, and $I D H$-WT GBM was 43.4, 42.6, 39 , 48.6, and 55.3 years, respectively. The male-tofemale ratio of these groups were 1:1, 7.4:2.6, 7:3, 4:6, and 6.6:3.4, respectively. Therefore, the ODG group had no gender predominance, but other subtypes had male predominance except for IDH-WT AA. Clinicopathological data were obtained from medical and pathological records, which are summarized in Table 1 . Representative MRI, hematoxylin and eosin (H\&E) features and immunohistochemical staining of IDH1 (H09) and ATRX are demonstrated in Figs. 1 and 2. This study followed the principles of the World Medical Association Declaration of Helsinki and it was approved by the Institutional Review Board of SNUH (IRB No.: 1307-093-505).

\section{Immunohistochemistry}

Formalin-fixed paraffin-embedded (FFPE) tissue blocks were cut into 3- $\mu \mathrm{m}$-thick slices and underwent immunohistochemistry (IHC). The DO-7 monoclonal antibody $(\mathrm{mAb})$ for p53 (1:1000, DAKO, Glostrup, Denmark), sc48817 for Olig2 (1:500, Santa Cruz Biotechnology, Santa Cruz, US), the anti-human Ki-67 mAb (1:1000, clone MIB-1, DAKO), ATRX (Merck (Sigma-Aldrich), St Louis, US), and the $\mathrm{mAb}$ against the $\mathrm{R} 132 \mathrm{H}$ mutation in $I D H 1$ (1:100, clone H09, Dianova, Heidelberg, Germany) were used for accurate pathological diagnosis (Table 2). The appropriate positive controls were used and primary antibodies were omitted as negative controls.

Virtual microscope scanning was performed on Ki-67-, p53-, and Olig2-immunostained slides using the Aperio Spectrum Plus image analyzer (Leica Biosystems Nussloch $\mathrm{GmbH}$, Nußloch, Germany) and we used them as an aid when reviewing the diagnosis. Intact tumor areas of each slide, excluding areas with crush artifacts, necrosis, and other poor-quality regions, were evaluated. For automated quantitation of each marker, nuclear V9 IHC algorithms of the Aperio Spectrum Plus image analyzer were applied after validating the data from comparisons to manual counts in a number of representative cases. The proportion of cells expressing Ki-67, p53, and Olig2 in the nuclei was calculated as a percentage of the total number of tumor cell-nuclei. We used a $30 \%$ cut off for p53 positivity.

\section{Fluorescence in situ hybridization for $1 p$ and $19 q$}

FFPE blocks underwent fluorescence in situ hybridization (FISH). Paired fluorescein isothiocyanate (FITC)/rhodamine-labeled DNA probes for chromosome regions 1p (LSI1p36/LSI1q25) and 19q (LSI19q13/ LSI19p13) were used for deletion analysis.

Green and red fluorescent signals were counted under a BX01 fluorescence microscope (Olympus, Olympus 
Table 1 Patients characteristics in our study cohort

\begin{tabular}{|c|c|c|c|c|c|c|}
\hline \multirow[t]{2}{*}{ Variable } & \multirow[b]{2}{*}{ No } & \multirow{2}{*}{$\begin{array}{l}\text { Group } 1 \\
\text { Oligodendroglioma, } \\
\text { IDH-mutant and } \\
1 \text { p19q-codeletion } \\
\text { WHO grade II \& III } \\
n(\%)\end{array}$} & \multirow{2}{*}{$\begin{array}{l}\text { Group } 2 \\
\text { Anaplastic } \\
\text { astrocytoma, } \\
\text { IDH-mutant, } \\
\text { WHO grade III } \\
n(\%)\end{array}$} & \multirow[t]{2}{*}{$\begin{array}{l}\text { Group } 3 \\
\text { Glioblastoma, } \\
\text { IDH-mutant, } \\
\text { WHO grade IV } \\
\text { n(\%) }\end{array}$} & \multirow{2}{*}{$\begin{array}{l}\text { Group } 4 \\
\text { Anaplastic } \\
\text { astrocytoma, } \\
\text { IDH-wildtype, } \\
\text { WHO grade III } \\
n(\%)\end{array}$} & \multirow{2}{*}{$\begin{array}{l}\text { Group } 5 \\
\text { Glioblastoma } \\
\text { IDH-wildtype } \\
\text { WHO grade IV } \\
n(\%)\end{array}$} \\
\hline & & & & & & \\
\hline Number of Patients & 168 & $65(100)$ & $23(100)$ & $13(100 \%)$ & $15(100 \%)$ & $52(100 \%)$ \\
\hline Age (years) & Mean (range) & $43.4(21-65)$ & $42.6(21-65)$ & $39(29-49)$ & $48.6(26-72)$ & $55.3(27-79)$ \\
\hline \multirow[t]{2}{*}{ Gender } & Male & $33(50.8)$ & $17(73.9)$ & $9(69.2)$ & $6(40)$ & $35(66.0)$ \\
\hline & Female & $32(49.2)$ & $6(26.1)$ & $4(30.8)$ & $9(60)$ & $17(34.0)$ \\
\hline Mean OS (mo) & & $38.0(0-95)$ & $59.4(0.5-114.8)$ & $40.0(11.8-72.3)$ & $28.2(0.5-76.9)$ & $19.6(0.5-116)$ \\
\hline Mean PFS (mo) & & $38.0(1-96)$ & $48.7(0.5-114.8)$ & $36.0(9.5-68.7)$ & $13.8(0.5-76.9)$ & $15.4(0-103)$ \\
\hline \multirow[t]{2}{*}{ TERT sequencing } & Wildtype & $2(3.1)$ & $22(95.6)$ & $3(23.1)$ & $12(80)$ & $11(21.2)$ \\
\hline & Mutant & $63(96.9)$ & $1(4.4)$ & $10(76.9)$ & $3(20)$ & $4178.8)$ \\
\hline \multirow[t]{2}{*}{ 1p, 19q FISH } & No co-deletion & $0(0)$ & $0(0)$ & $13(100)$ & $15(100)$ & $53(100)$ \\
\hline & Co-deletion & $65(100)$ & $23(100)$ & $0(0)$ & $0(0)$ & $0(0)$ \\
\hline ATRX $\Vdash H C$ & $\begin{array}{l}\text { Wildtype (retained) } \\
\text { Mutant (loss) }\end{array}$ & $\begin{array}{l}0(0) \\
65(100)\end{array}$ & $\begin{array}{l}7(30.4) \\
16(69.6)\end{array}$ & $\begin{array}{l}4(30.7) \\
9(69.2)\end{array}$ & $\begin{array}{l}9(60) \\
6(40)\end{array}$ & $\begin{array}{l}48(92.3) \\
4(7.7)\end{array}$ \\
\hline \multirow[t]{2}{*}{ MGMT MSP } & No methylation & $11(16.9)$ & $9(39.1)$ & $3(23.1)$ & $7(46.7)$ & $26(50.0)$ \\
\hline & Methylation & $54(83.1)$ & $14(60.9)$ & $10(76.9)$ & $8(53.3)$ & $26(50.0)$ \\
\hline Ki-67 labeling index (\%) & Mean (range) & $10.6(1.5-39.5)$ & Not available & $14(1-39.3)$ & Not available & $20.6(0-54.8)$ \\
\hline
\end{tabular}

Abbreviations: mo month, OS overall survival, PFS progression free survival, FISH fluorescent in situ hybridization, IHC immunohistochemistry, MSP methylation-specific PCR, HD homozygous deletion, WHO World Health Organization
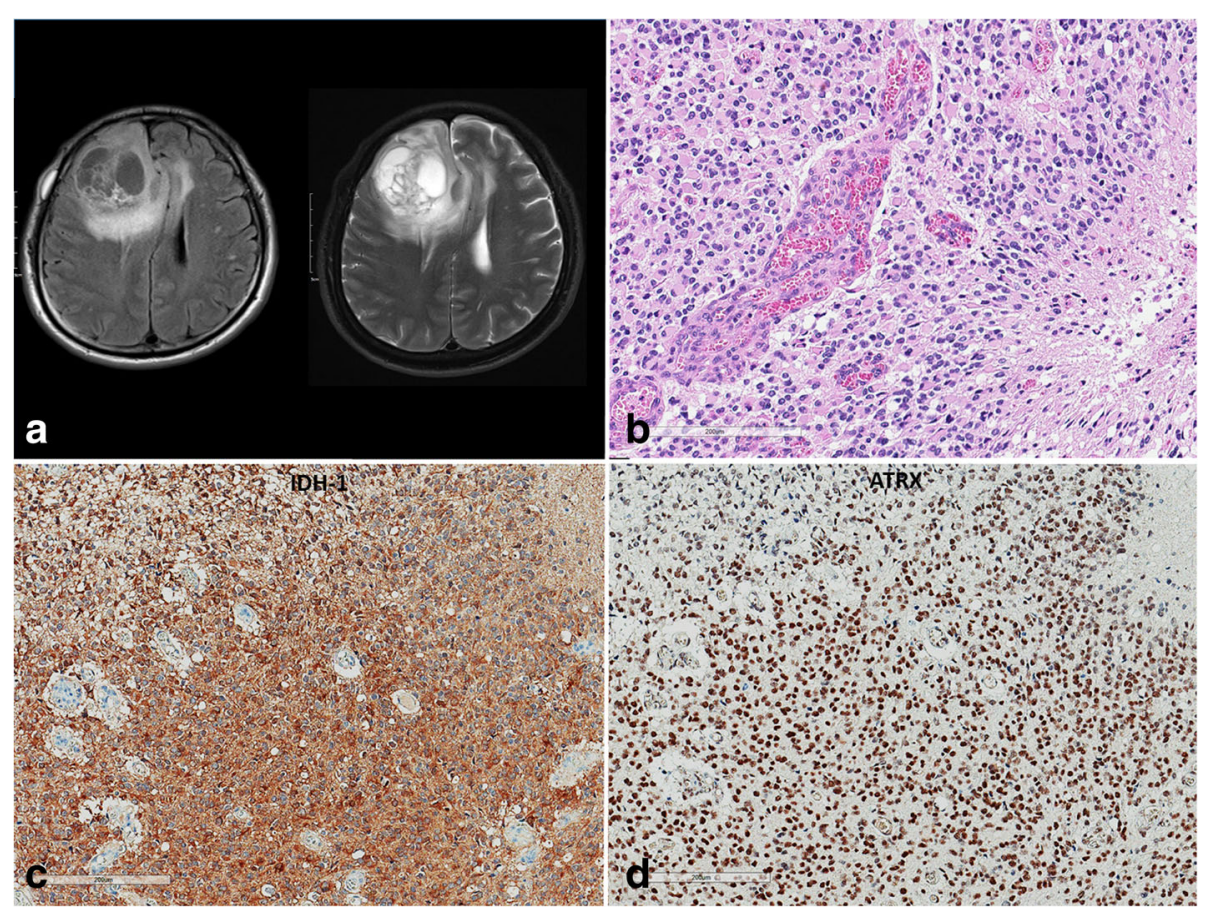

Fig. 1 a) MRI showing a solid and cystic mass involving the right frontal lobe with diffuse increased T2 signal intensity in the right frontal lobe, insula, and corpus callosum genu with subfalcine herniation on the left side. b) The tumor is indicative of glioblastoma by the presence of necrosis and microvascular proliferation. c, d) Tumor cells are positive for IDH-1 (H09), R132H-mutant, and retained expression of ATRX 


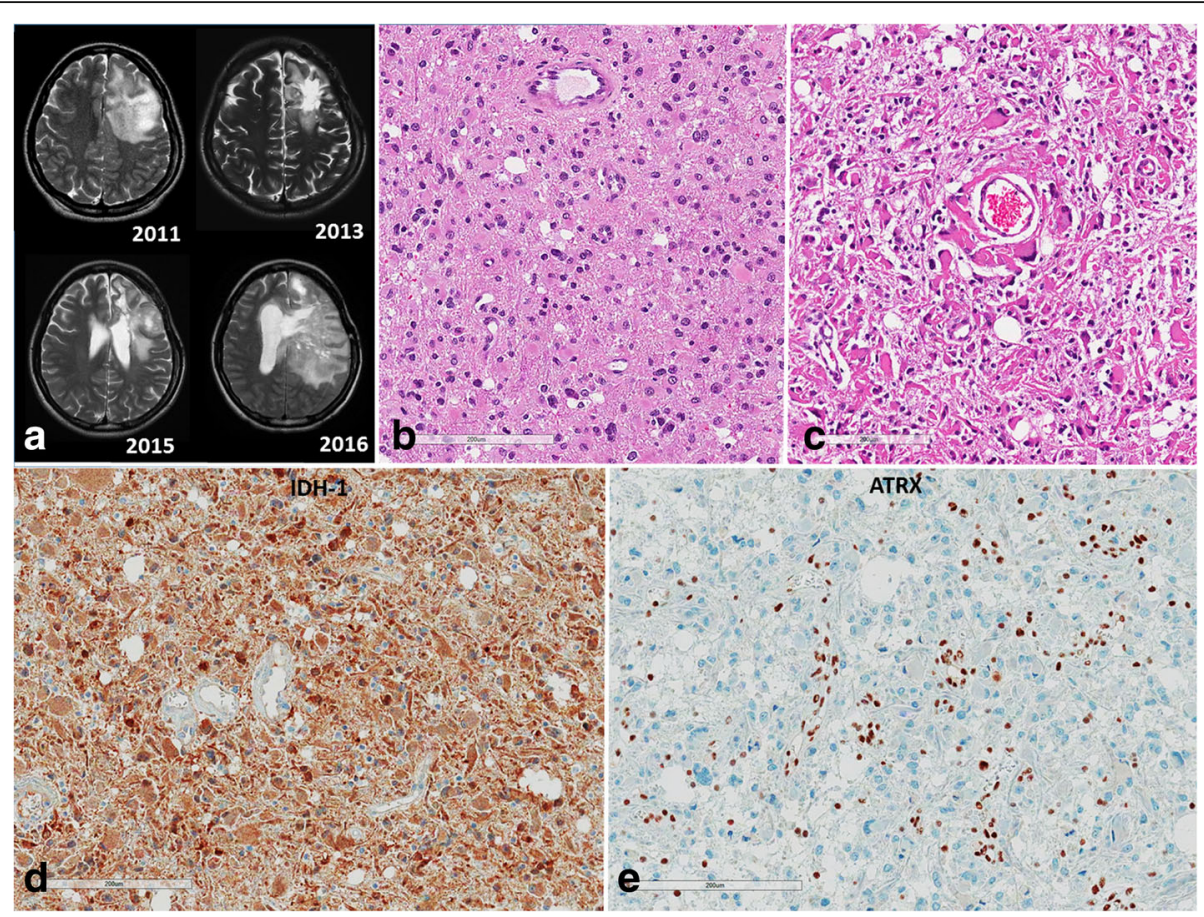

Fig. 2 Recurrent anaplastic astrocytoma, IDH-mutant. a) MRI showing initial tumor and 3 times recurrent tumors despite concurrent chemotherapy and radiotherapy. b) Initial tumor showing a sheet of neoplastic gemistocytic astrocytes with mild to moderate nuclear atypia, WHO grade III. c) The second recurrent tumor shows more pleomorphic nuclei with bizarre tumor cell features than those of initial tumor, but there is neither tumor necrosis nor microvascular proliferation. c, d) However, note IDH-1 and ATRX mutation were sustained (c: IDH1 (HO9) staining, d: ATRX immunostaining)

corporation, Japan) using the MetaMorph ${ }^{\odot}$ Imaging System (Universal Imaging, Molecular Devices, US). For each hybridization, the number of green and red signals was assessed using a minimum of 100 non-overlapping nuclei. An interpretation of a deletion was made when $>50 \%$ of nuclei harbored a single red or green signal. This determination was based on the frequency of nonneoplastic nuclei that contained one signal (median \pm 3 standard deviations) using the same probes in nonneoplastic control (seizure-resection) specimens.

\section{IDH1/IDH2 and TERT mutation analysis}

Sanger sequencing was used to determine the frequency of mutations in IDH1, IDH2, and TERT. Primer design

Table 2 Primary antibodies used in this study

\begin{tabular}{llll}
\hline Name & Manufacturer & Clone & Dilution \\
\hline $\begin{array}{l}\text { Glial fibrillary } \\
\text { acid protein }\end{array}$ & DAKO, Glostrup, Denmark & $6 F 2$ & $1: 300$ \\
Olig2 & $\begin{array}{l}\text { Santa Cruz Biotechnology, Santa Cruz, } \\
\text { US }\end{array}$ & sc48817 & $1: 500$ \\
P53 & DAKO, Glostrup, Denmark & DO7 & $1: 1000$ \\
Ki-67 & DAKO, Glostrup, Denmark & MIB-1 & $1: 1000$ \\
IDH1 & DIANOVA, Hamburg, Germany & DIAH09 & $1: 100$ \\
ATRX & MERCK (Sigma), St Louis, USA & HPA001906 & $1: 100$ \\
\hline
\end{tabular}

For antigen retrieval, cell conditioning with CC1 buffer pH 8.4 (Ventana, Export, PA) was used for all antibodies was based on sequence data from accession numbers NM 005896 for IDH1 and NM 002168 for IDH2 (http://www.ncbi.nlm.nih.gov). Representative H\&E stained slides were reviewed by two pathologists ( $\mathrm{SH}$ Park and JK Won), and a selected tumor region was macrodissected manually from consecutive FFPE sections. After deparaffinization, genomic DNA was extracted and underwent nested-PCR for IDH1 (186 bp), IDH2 (302 bp), and the TERTp. PCR primer sequences used for $I D H 1$ and $I D H 2$ are summarized in Table 2. PCR amplification and Sanger sequencing was performed using an ABI-PRISM 3730 DNA Analyzer (Applied Biosystems, Vernon Hills, IL, USA). PCR was performed in $40 \mu \mathrm{L}$ reaction conditions that included standard buffer conditions, $200 \mathrm{ng}$ of DNA, and GoTaq DNA Polymerase (Promega, Madison, WI, USA). PCR amplification for IDH1 consisted of 45 cycles with denaturation at $95{ }^{\circ} \mathrm{C}$ for $30 \mathrm{~s}$, followed by annealing at $62{ }^{\circ} \mathrm{C}$ for $30 \mathrm{~s}$, and extension at $72{ }^{\circ} \mathrm{C}$ for $1 \mathrm{~min}$, whereas PCR amplification for IDH2 consisted of $40 \mathrm{cy}-$ cles with denaturing at $95{ }^{\circ} \mathrm{C}$ for $30 \mathrm{~s}$, followed by annealing at $55{ }^{\circ} \mathrm{C}$ for $30 \mathrm{~s}$, and extension at $72{ }^{\circ} \mathrm{C}$ for 1 min. Two microliters of PCR amplification product were sequenced using the BigDye Terminator v3.1 Cycle Sequencing Kit (Applied Biosystems, Foster City, CA, USA). Twenty-five cycles were performed using $12 \mathrm{ng}$ of the sense primers IDH1f $5^{\prime}$-M13-GTAAAACGACG 
GCCAGTCGGTCTTCAGAGAAGCCA-3' or IDH2f 5'-GCTGCAGTGGGACCACTATT-3' with denaturation at $96{ }^{\circ} \mathrm{C}$ for $10 \mathrm{~s}$, annealing at $50{ }^{\circ} \mathrm{C}$ for $5 \mathrm{~s}$, and extension at $60{ }^{\circ} \mathrm{C}$ for $4 \mathrm{~min}$.

Two hotspot mutations, C228T and C250T, in the TERTp were screened using the oligonucleotide primers shown in Table 3. M13 has a universal sequencing primer site with the sequence 5'-GTAAAACGACGGC CAGT-3' that was used for PCR amplification of the proximal TERTp for Sanger sequencing using standard methods. PCR was performed in $50 \mu \mathrm{L}$ reaction mixtures containing $5 \mu \mathrm{L}$ of DNA, $10 \mathrm{mM}$ of each dNTP, 10 pmole $/ \mu \mathrm{L}$ each primer, $5 \mathrm{X}$ Band Doctor $^{\mathrm{rw}}$, $10 \mathrm{X}$ h-Taq Reaction buffer (15 mM $\mathrm{MgCl}_{2}$ mixed), and $2.5 \mathrm{U} / \mu \mathrm{L}$ of Solg $^{\text {tix }} \mathrm{h}$-Taq DNA Polymerase. PCR was initiated at $95{ }^{\circ} \mathrm{C}$ for $15 \mathrm{~min}$, followed by 45 cycles of $95^{\circ} \mathrm{C}$ for $30 \mathrm{~s}, 62{ }^{\circ} \mathrm{C}$ for $30 \mathrm{~s}$, and $72{ }^{\circ} \mathrm{C}$ for $1 \mathrm{~min}$ with a final extension of $72{ }^{\circ} \mathrm{C}$ for $7 \mathrm{~min}$.

\section{Methylation-specific PCR}

Methylation status of the O-6-methylguanine-DNA methyltransferase (MGMT) gene promoter was determined by extracting tumor genomic DNA from FFPE sections for bisulfite conversion using the EZ DNA methylation-Gold Kit (Zymo Research, Orange County, CA, USA). MGMT methylation-specific PCR was performed using primer pairs specific to methylated and unmethylated MGMT promoter sequences (Table 3).

\section{Statistical analysis}

All statistical analyses were performed using SPSS software v23 (IBM Corp., New York, NY, USA). Comparisons between variables were performed using the $\chi^{2}$ test, Fisher's exact test, or the Student's t-test. Disease-free survival (DFS) was measured from the date of surgery to that of disease recurrence or onset of metastasis. OS was measured from the date of diagnosis until death from any cause. Survival analysis was performed using the Kaplan-Meier method with log-rank test. Multivariate Cox regression analysis was performed with consideration of co-linearity. Two-sided $P$-values $<0.05$ were considered statistically significant.

\section{Results}

TERTp mutation status and relationship with clinicopathological parameters

Somatic TERTp mutations were analyzed by direct Sanger sequencing. TERTp mutations were detected in $96.9 \%$, $4.4 \%, 76.9 \%, 20.0 \%$ and $84.6 \%$ of patients in Group 1, 2, 3, 4 , and 5, respectively. The C228 T mutation was much more frequent than the C250T mutation in our cohort (Fig. 3 and Table 4). There was no evidence of an association between TERTp mutation and IDH or ATRX mutation (ATRX loss) or MGMTp methylation status, gender preference, or age preference in all patient groups except the patients with AA (grade III) by Pearson $\chi^{2}$ test. Further, in patients with AA (combined groups 2 and 4), the TERTp mutation was more common for over 55 years of age $(P=0.004)$, which may be the reason why TERTp mutation is an adverse marker specifically for IDH-WT AA. In this study we could not reach statistical significance $(P$ $=0.113$ ), because of the lack of the number of TERTp mutant cases (Fig. 5c).

\section{IDH1/IDH2 mutation status and relationship with clinicopathological parameters}

The $\mathrm{R} 132 \mathrm{H}$ mutation in IDH1 was found in 60.5\% (23/38) of patients with AA and $20.0 \%(13 / 65)$ of our patients with GBM (Groups 3 and 5), whereas all patients with ODG (Group 1) had a mutation either in IDH1 $(n=62)$ or IDH2 $(n=3)$, as determined by Sanger sequencing. Correlation analysis between $I D H$ mutation and clinicopathological features in these five groups of gliomas revealed that $I D H$ mutation was associated with a younger age at diagnosis and in patients with MGMT methylation and ATRX mutation, respectively by Pearson $X^{2}$ test, but it was not correlated with TERTp mutation $(p<0.05)$.

MGMTp methylation and ATRX mutation (ATRX loss) status MGMTp methylation was present in $83.1 \% 60.9 \%$, $76.9 \%, 53.3 \%$, and $49.1 \%$ of cases from Groups 1, 2, 3, 4, to 5, respectively. An ATRX mutation was present in $69.6 \% 69.2 \%, 40.0 \%$, and $5.7 \%$ of patients from Group 2, 3 , 4, to 5 respectively, but it was not found in patients with ODGs (Group 1). MGMTp methylation was more

Table 3 Primer sequences of IDH1/IDH2, TERT promoter, and MGMT for Sanger sequencing

\begin{tabular}{llll}
\hline & Prime & & \\
\cline { 2 - 3 } Gene & Forward & Reverse & Product (bp) \\
\hline IDH1 & 5'-M13-GTA AAA CGA CGG CCA GTC GGT CTT & 5'-GCG GAT AAC AAT TTC ACA CAG GGC \\
IDH2 & AAG AGA AGC CA-3' & AAC ACA TTA TTG C-3' & 180-190 \\
TERT & 5'-GCT GCA GTG GGA CCA CTA TT-3' & 5'-TGT GGC GTT GTA CTG CAG AG-3' & 295-305 \\
& 5'-M13-GTA AAA CGA CGG CCA GTC ACC CGT & 5'-GCA CCT CGC GGT AGT GG-3' & \\
MGMT & CCT GCC CCT TCA CCT T-3' & & $300-310$ \\
\hline
\end{tabular}




\section{a $\begin{array}{llll}40 & 50 & 60 & 60\end{array}$ TCCCGACCC CT CCCGGGTCC CAGCCCCCTCCGGGCCCT CCC}

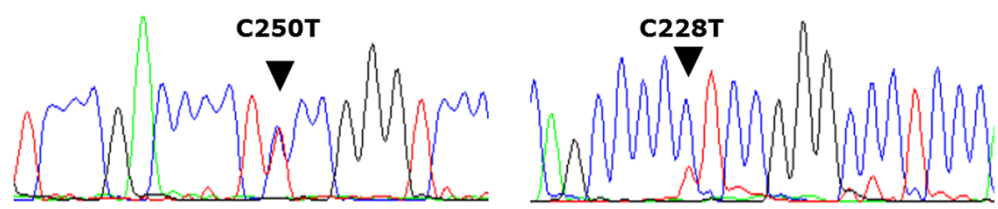

Fig. 3 Electropherograms showing sequence of the TERT promoter region with the two hot-spot mutations a) C250T and b) C228T

common in $I D H$-mutant GBMs, but was not associated with IDH mutation status in AA. ATRX mutation was also more common in IDH-mutant GBMs and/or younger patient under 55 years old with GBM.

\section{Prognostic impact of TERTp, ATRX, and IDH mutations, and MGMTp methylation}

Using Kaplan-Meier survival analysis, we found that these five groups were well segregated $(P=0.000)$ (Fig. 4a) and patients with IDH-mutant gliomas had significantly better survival compared to those with IDH-WT gliomas $(P<0.001, P<0.003)$ (Fig. 4b and c).

In addition, we found that MGMTp methylation was a good prognostic factor in pooled groups with total GBM (Groups 3 and 5$)(P=0.008)$ and total AA and GBM groups (groups 2, 3, 4, and 5 combined) $(P=0.017)$ (Fig. 4d); however, in individual groups of gliomas, MGMTp methylation was not correlated with OS. In $I D H$-mutant and IDH-WT GBMs (Groups 3 and 5), we found that MGMTp methylation was a borderline indicator of better prognosis $(P=0.051$ and 0.076) (Fig. 4e-f). In total AA (Groups 2 and 4), MGMTp methylation was not correlated with survival $(P=0.164)$.

In group 1 (ODG), we found that TERTp mutations were not associated with either OS or PFS $(P=0.688$

Table 4 The frequency of TERTp mutations

\begin{tabular}{llll}
\hline Diagnosis & $\begin{array}{l}\text { C228T } \\
\text { mutation }\end{array}$ & $\begin{array}{l}\text { C250T } \\
\text { mutation }\end{array}$ & $\begin{array}{l}\text { Both TERT } \\
\text { mutations }\end{array}$ \\
& No. (\%) & No. (\%) & No. (\%) \\
\hline Oligodendroglioma & $55 / 63(87.3)$ & $8 / 63(12.7)$ & $63 / 65(96.9)$ \\
AA, IDH-mutant & $1 / 1(100)$ & $0 / 1(0)$ & $1 / 23(4.4)$ \\
GBM, IDH-mutant & $7 / 10(70)$ & $3 / 10(30)$ & $10 / 13(76.9)$ \\
AA, IDH-wildtype & $2 / 3(66.7)$ & $1 / 3(33.3)$ & $3 / 15(20.0)$ \\
GBM, IDH-wildtype & $36 / 44(81.8)$ & $8 / 44(18.2)$ & $44 / 52(84.6)$ \\
\hline
\end{tabular}

Abbreviations: $A A$ anaplastic astrocytoma, GBM glioblastoma, TERTp, TERT promoter region and $P=0.427$, respectively) (Fig. $5 \mathrm{a}$ ). In a combined cohort with Groups 2 and 4, TERTp mutation was strongly associated with patient survival (Fig. 5b), although this finding may be attributed to the TERTp mutation being more frequent in the $I D H$-WT AA group, which has the worse prognosis than $I D H$-mutant AA.

In Group 4 (IDH-WT AA), we found that patients with TERTp-mutant tumor showed shorter median survival compared to patients with TERTp-WT tumors [median survival with 95\% CI: 8.0 months (0.00020.002) vs. 18.1 months (0.000-46.107), respectively]; however, TERTp mutation status did not have an statistically significant effect on OS or PFS (Table 5, Fig. 5c and d), possibly because the number of TERTp mutant cases were too few to attain statistical significance $(P=0.113)$. When we evaluated the prognostic value of TERTp mutations in each of the five groups by Cox regression analysis, we found no evidence of association, with the exception of Group 5 (IDH-WT GBM), in which we found by multivariate analysis that patients with TERTp mutation had statistically worse prognosis than patients with TERTp-WT $(P=0.045)$ [median survival with $95 \%$ CI: 16.4 months (13.012-19.516) vs. 13.0 months (1.021-25.039), respectively] (Table 5). In addition, we found that having an ATRX mutant tumor did not affect patient OS or PFS in any of the five groups (all $P>0.05$ ) (Fig. 6a and b).

From Kaplan Meier survival analysis of diffuse astrocytic tumors of grade III and IV, we found that IDH-mutant and younger age-patients (under 55 years of age) (Figs. 4b-c and 6d) were associated with statistically significant better survival compared to their counter parts. In the IDH-mutant groups (Group 2 and Group 3), because most patients were younger than 55 years of age, age was not a factor for patient survival (Table 1) and particularly in GBMs (combined group 3 and group 5), the $I D H$-mutation was exclusively present in the patients younger than 55 years old.

On multivariate Cox regression analysis of the Group 5 (IDH-WT GBMs), TERT-mutant $(P=0.045)$, MGMTp 


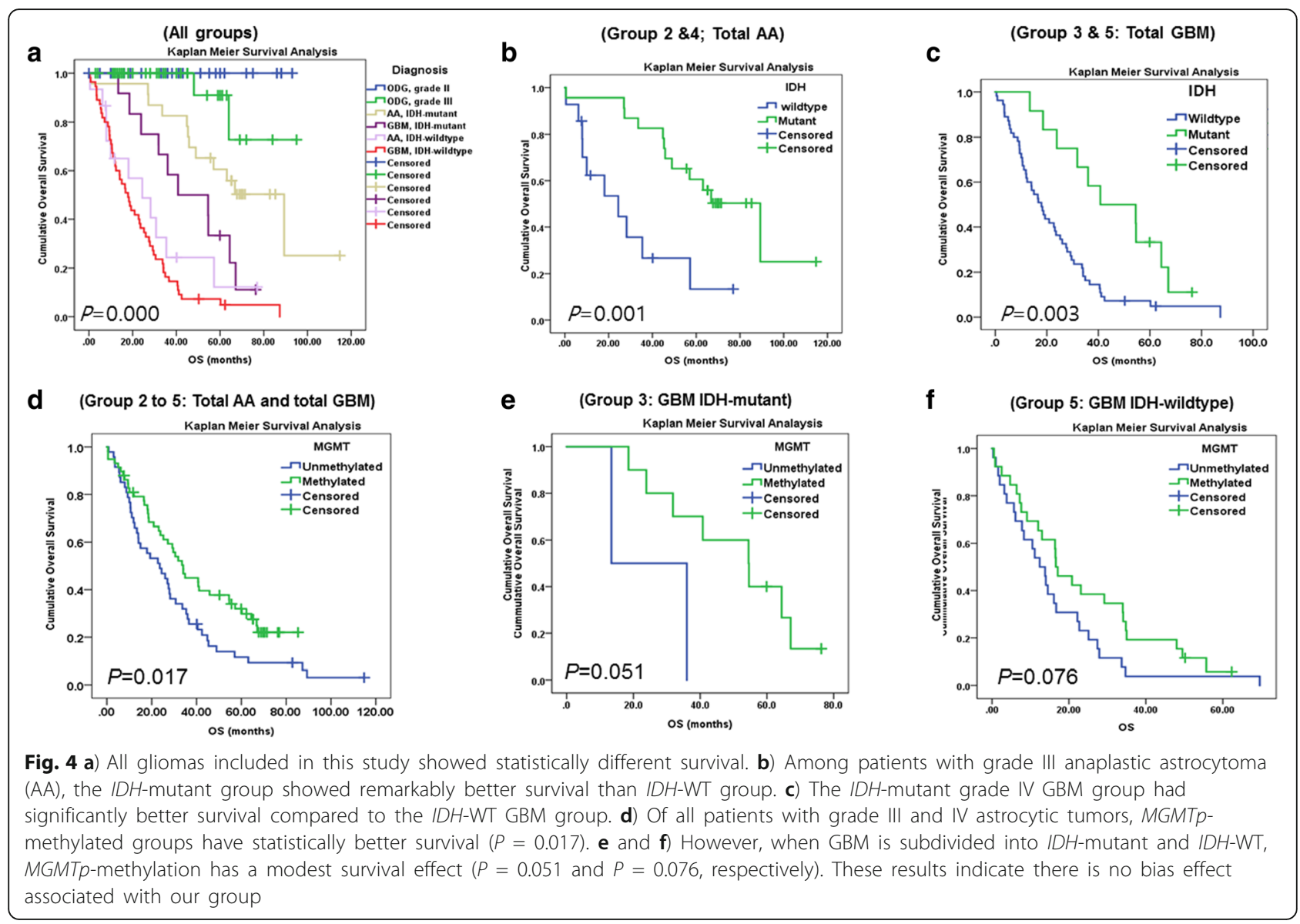

methylation $(P=0.003)$ and younger age under 55 years old $(P=0.001)$ were statistically significant good prognostic factors (Table 5), but in other groups, patients with TERTp mutation, ATRX mutation, MEMTp methylation and younger age did not show significantly different survival rate than counter parts. Regarding agerelated factors, no evidence of statistical significance was found because of the small number of patients aged 55 years or older with Type III astrocytomas (Fig. 6c).

\section{Discussion}

Telomerase is a specialized reverse transcriptase that maintains telomere length [10]. Telomerase activity is robustly expressed in embryonic cells, while it is suppressed in fully matured somatic cells during adult life. However, it is expressed in approximately $85 \%$ of solid tumors and most immortalized cell lines. Recently, several studies have reported that TERTp mutations are frequently found in gliomas, especially in ODGs and GBMs, which results in altered telomere lengthening and lead to prolonged longevity of tumor cells by escaping from the tumor cell senescence [3]. Aita et al. [19] found that TERTp mutations are present in $>70 \%$ of patients with ODG and GBM, and that the frequency of
TERTp mutation is even higher than previous reports in ODG and GBM, because the diagnostic criteria of diffuse glioma became more strict with the integration of genetics in the diagnosis according to the revised 2016 WHO classification criteria.

Based on this finding, we studied the frequency and putative prognostic importance of mutations in TERTp and $A T R X$ as well as MGMTp methylation in five patient groups, which were classified by 2016 revised WHO classification of CNS tumors: Group 1: ODGs, Group 2: grade III AA (IDH-mutant), Group 3: grade IV GBM (IDH-mutant), Group 4: grade III AA (IDH-WT), and Group 5: grade IV GBM (IDH-WT) [19]. These five groups were well-classified on the basis of OS rate by Kaplan Meier Survival analysis (Fig. 4a). Patients with $I D H$-mutant GBM showed better survival compared to those with $I D H$-WT AA and IDH-WT GBM; however, they showed worse OS than patients with $I D H$-mutant AA. The OS of patients with $I D H$-WT AA was comparable to that found in patients with $I D H$-WT GBM. These findings verified our cohort was not deviated groups.

Furthermore, we found that the TERTp mutation frequencies in these five groups were $96.9 \%, 4.4 \%, 76.9 \%$, $20 \%$, and $84.6 \%$, respectively (Table 4). Therefore, 


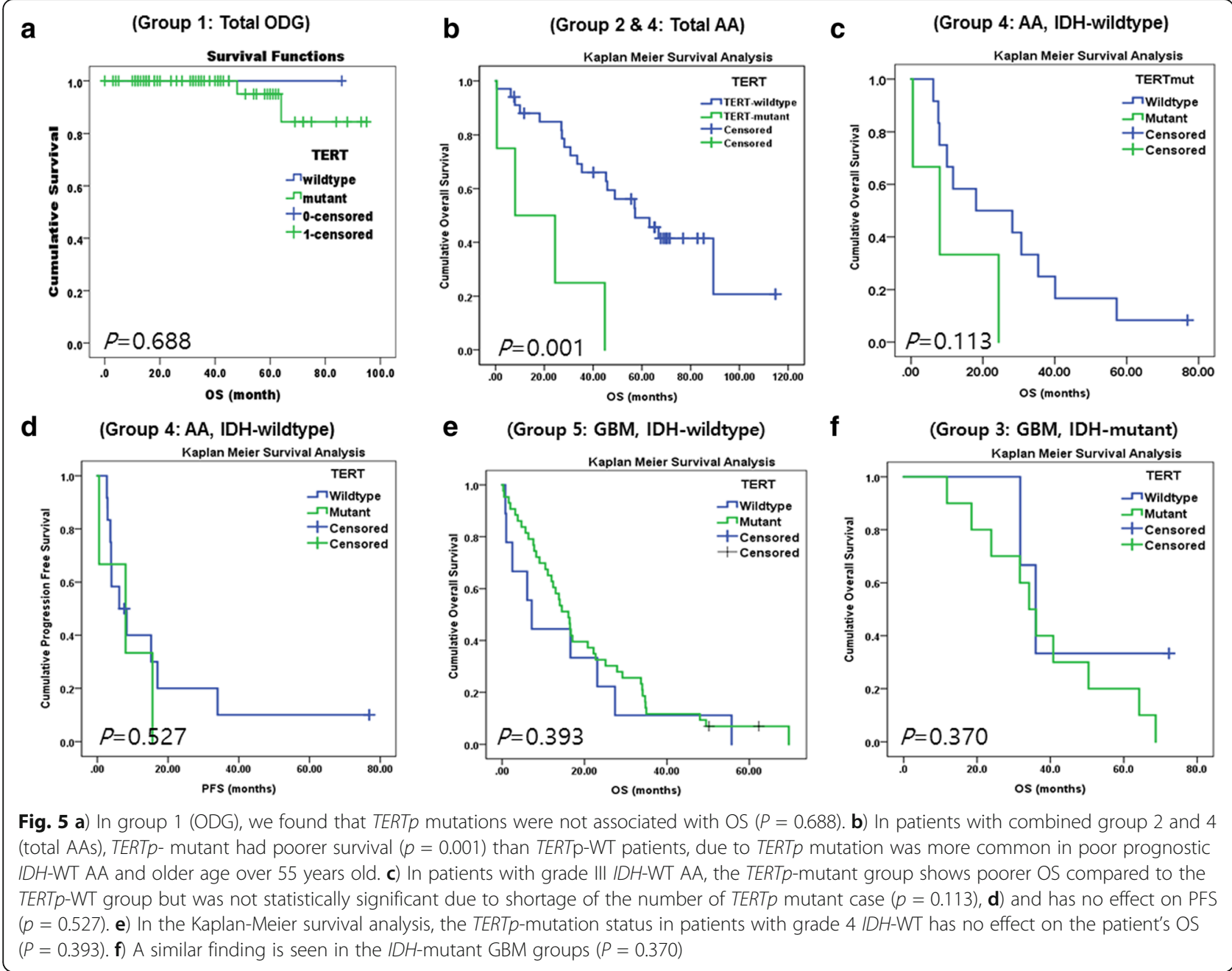

patients with grade III $I D H$-mutant AA (Group 2) had the lowest incidence of TERTp mutation (4.4\%) and patients with IDH-WT grade III AA (Group 4) had the second lowest frequency of TERTp mutation (20\%). These frequencies about half of those reported by EckelPassow et al. [9]. Among their series of grade II or III gliomas $(N=586), 40.4 \%(40 / 99)$ of IDH-WT astrocytoma and $10.1 \%$ (31/306) of IDH-mutant astrocytoma was TERTp-mutated tumors and remained 181 cases were triple positive $(1 \mathrm{p} / 19 \mathrm{q}$ co-deleted, $I D H$-mutant, and TERT-mutated) ODG [9]. TERT mutation only cases (that means IDH-WT astrocytomas) were the worst prognostic group and that group had worse survival than that of patient with $I D H$-WT and TERT-WT [9].

In addition, we found increased frequencies of TERTp mutations in patients with ODGs and GBMs, both $I D H$ mutant and $I D H$-WT, which is comparable to those found in a previous report [14]. Therefore, similar to previously reported studies, we propose that telomerase activation may be an underlying mechanism in these gliomas, especially in cases of ODG and GBMs [5]. In addition, we found that occurrence of the $\mathrm{C} 228 \mathrm{~T}$ and C250T mutations were mutually exclusive in our cohort, and that C228T was more common than C250T (Table 4) $[21]$.

In previous studies, the $1 \mathrm{p} / 19 \mathrm{q}$ co-deletion was strongly associated with mutations in TERTp [13, 25]. In the present study, we found that $96.9 \%$ of patients with ODG had a TERTp mutation, whereas two patients with ODG were TERTp-WT (Table 4). However, in cases with GBM that did not harbor the $1 \mathrm{p} / 19 \mathrm{q}$ co-deletion, we found that they had a high frequency of TERTp mutation, and therefore, conclude that the TERTp mutation is not exclusively associated with the $1 \mathrm{p} / 19 \mathrm{q}$ co-deletion.

Regarding TERTp mutation from a prognostic perspective in diffuse gliomas, previous studies showed conflicting results. Labussiere et al. found that TERTp mutations may be associated with poorer outcome in high-grade gliomas, [16] however, Pekmezci et al. reported that TERT-mutants had significantly worse survival only in IDH-WT astrocytoma, which includes 
Table 5 The result of multivariate analysis on group 4 and 5

\begin{tabular}{|c|c|c|c|c|c|c|c|c|c|}
\hline \multirow[t]{2}{*}{4} & \multirow{2}{*}{$\begin{array}{l}N \\
(\%)\end{array}$} & \multirow{2}{*}{$\begin{array}{l}\text { Age median } \\
\text { (range) }\end{array}$} & \multirow{2}{*}{$\begin{array}{l}\text { Median survival years (95\% } \\
\mathrm{Cl})\end{array}$} & \multicolumn{3}{|c|}{ Univariate analysis } & \multicolumn{3}{|c|}{ Multivariate analysis } \\
\hline & & & & Hazard ratio & $95 \% \mathrm{Cl}$ & $p$ Value & Hazard ratio & $95 \% \mathrm{Cl}$ & $p$ Value \\
\hline \multicolumn{10}{|l|}{ Group 4} \\
\hline AA, IDH-wildtype & 15 & $51(26-72)$ & $18.1(0.000-36.278)$ & & & & & & \\
\hline TERTp-mutant & 3 & $69.0(62-72)$ & $8.0(0.000-20.002)$ & 0.341 & $0.85-1.377$ & 1.113 & 1.11 & $0.116-10.608$ & 0.928 \\
\hline TERTp-wildtype & 12 & $46.5(27-58)$ & $18.1(0.000-46.107)$ & & & & & & \\
\hline ATRX-mutant & 9 & $44.5(27-69)$ & $28.2(0.954-55.446)$ & 0.555 & $0.182-1.687$ & 0.299 & 0.731 & $0.214-2.498$ & 0.618 \\
\hline ATRX-wildtype & 6 & $54(41-72)$ & 11.7 (6.733-16.667) & & & & & & \\
\hline MGMTp-methylated & 8 & 48.5926-62) & $28.2(18.448-37.952)$ & 1.109 & $0.365-3.367$ & 0.856 & 1.076 & $0.261-4.434$ & 0.919 \\
\hline $\begin{array}{l}\text { MGMTp- } \\
\text { unmythylated }\end{array}$ & 7 & $54(27-72)$ & $7.9(2.218-13.582)$ & & & & & & \\
\hline Age $<55$ years old & 10 & $\begin{array}{l}41.5(26- \\
54.9)\end{array}$ & $28.2(8.676-47.724)$ & 4.027 & $1.048-15.472$ & 0.043 & 0.296 & $0.042-2.107$ & 0.224 \\
\hline Age $>55$ years old & 5 & $62(57-69)$ & $8.0(7.785-8.215)$ & & & & & & \\
\hline \multicolumn{10}{|l|}{ Group 5} \\
\hline GBM, IDH-wildtype & 52 & $55(17-79)$ & $14.5(10.378-18.622)$ & & & & & & \\
\hline TERTp-mutant & 41 & $58(36-79)$ & $16.4(13.012-19.516)$ & 0.768 & $0.390-1.513$ & 0.445 & 2.111 & $1.016-4.389$ & 0.045 \\
\hline TERTp-wildtype & 11 & $42(31-69)$ & $13.0(1.021-25.039)$ & & & & & & \\
\hline ATRX-mutant & 5 & $58(29-69)$ & $13.0(0.513-25.547)$ & 0.682 & $0.269-1.733$ & 0.422 & 1.269 & $0.499-3.231$ & 0.617 \\
\hline ATRX-wildtype & 47 & $55(31-79)$ & $16.4(12.773-20.027)$ & & & & & & \\
\hline MGMTp-methylated & 26 & $57.5(29-79)$ & $18.3(12.671-23.989)$ & 0.579 & $0.326-1.031$ & 0.061 & 0.665 & $1.399-5.078$ & 0.002 \\
\hline $\begin{array}{l}\text { MGMTp- } \\
\text { unmythylated }\end{array}$ & 26 & $51(36-79)$ & $13.0(9.407-16.653)$ & & & & & & \\
\hline Age $<55$ years old & 24 & $45(29-54.9)$ & $20.8(13.599-28.001)$ & 1.843 & $1.047-3.331$ & 0.035 & 3.090 & $1.578-6.049$ & 0.001 \\
\hline Age $>55$ years old & 28 & $67(55.3-79)$ & $10.5(5.703-15.297)$ & & & & & & \\
\hline
\end{tabular}

grades II and III [23]. Such contradictory effects of TERTp mutation on patient outcome between groups have been reported previously [9]. Abudumijiti et al. found comparable results to those of Pekmezci et al. and concluded that adult IDH-WT lower-grade gliomas should be further classified by TERTp mutation status [1]. Similarly, our detailed analysis using group classifications according to $\mathrm{IDH}$-mutation status and multivariate analysis revealed that only in those cases with grade IV IDH-WT GBM, TERTp-mutation was associated with worse OS compared to TERTp-WT (Table 5). We did not study grade II diffuse astrocytoma because we wanted a homogeneous group of grade III cases and to not be potentially biased by grading. In $I D H$-mutant AA, the number of TERTp-mutant cases was too small to evaluate its effect, while in grade IV GBMs, regardless of IDH-mutation status, TERTp-mutation did not affect patient OS (Fig. $5 \mathrm{c}$ and d). However, it was difficult to verify this finding in patients with ODG because of the high frequency of TERTp mutations (96.9\%) in this tumor group. In addition, we found that the MGMTp methylation group had better survival in combined grade III and IV astrocytic tumors and in both $I D H$-mutant and $I D H$ -
WT GBMs; however, we found that ATRX mutation did not confer a prognostic effect in any of the five groups evaluated in our study, which is different from Pekmezci et al.'s conclusion [23].

Our findings differ from those of Eckel-Passow et al. [9]. They concluded that the patients with grade II or III gliomas with TERTp mutation only revealed the worst survival, but TERTp mutation status was not correlated with survival rate of patients with grade IV glioma. This result means TERTp mutation may be poor prognostic marker only in IDH-WT grade II or III astrocytomas.

Further studies incorporating such classification using molecular alterations including TERTp mutations are warranted to determine their putative prognostic or diagnostic importance for gliomas.

Because cancer cells acquire immortality by telomere lengthening, the telomerase enzyme is a likely anticancer therapeutic target [10, 24]. Daniel et al. [24] proposed that the human immunodeficiency virus reverse transcriptase inhibitor azidothymidine (AZT) acts as a telomerase inhibitor. In addition, several telomerasetargeting inhibitors such as the antisense oligonucleotide inhibitor GRN163L and immunotherapies that use 
a

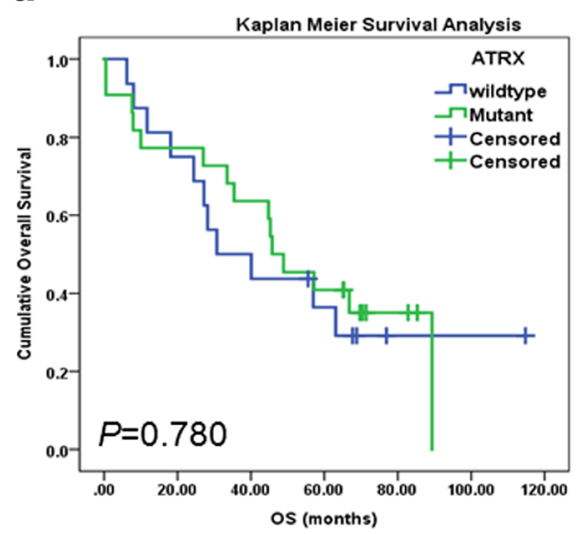

C (Group 4: AA, IDH-wildtype)

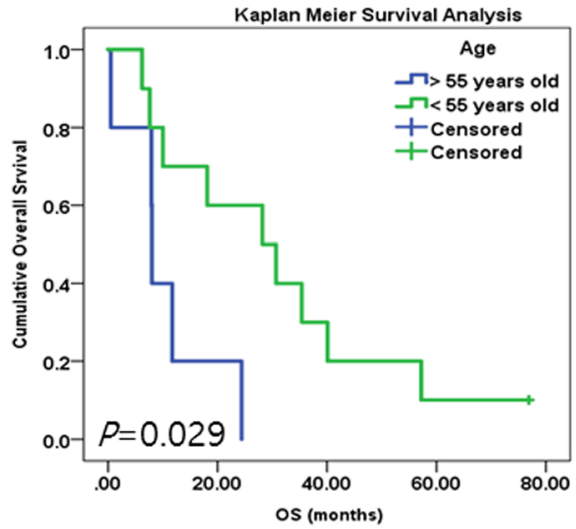

b

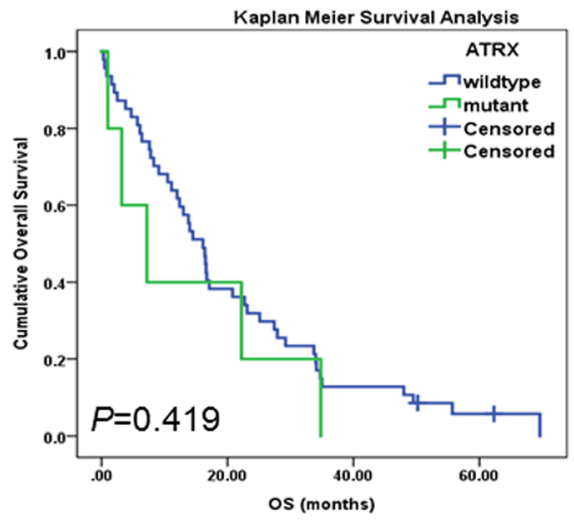

d (Group 3 \& 5: GBM IDH-wildtype)

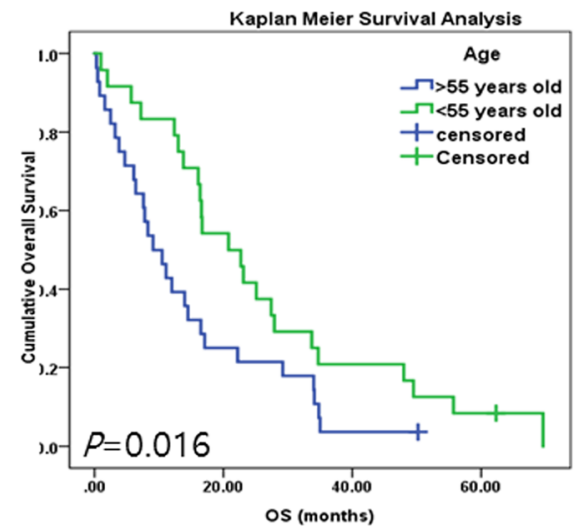

Fig. 6 ATRX mutation was not a statistically significant biomarker in all grade III and IV astrocytic tumors. a) In cases with grade III anaplastic astrocytoma, combined IDH-mutant and -WT tumors, the ATRX-mutant group does not show statistically different OS compared to the ATRX-WT group $(P=0.780)$, b) a finding which is comparable to that found in the GBM IDH-WT group $(P=0.419)$. $\mathbf{c}$, $\mathbf{d})$ Statistical significance of age were obtained in the group 4 (AA, IDH-wildtype) $(P=0.029)$, and the group 5 (IDH-WT GBMs) $(P=0.016)$, patients older than 55 years have a statistically significant worse OS compared to patients younger than 55 years of age

dendritic cells (GRVAC1), the hTERT peptide (GV1001), or cryptic peptides (Vx-001) are under investigation [24].

\section{Conclusion}

In conclusion, mutations in the promoter of TERTp are common in patients with GBM or ODG. In terms of survival effect, mutations in IDH1 and MGMTp methylation and age under 55 were associated with favorable prognosis in diffuse gliomas and in cases with grade III AA (combined IDH-mutant and $I D H$ WT AA), TERTp mutant carriers had worse OS than TERTp WT carriers. In multivariate analysis, TERTp mutation was strongly correlated with poor outcome in patients with IDH-WT GBM, suggesting that it may be of prognostic value in this subgroup of patients with gliomas.

In ODGs, because all tumors have a TERTp mutation except for two casess, we could not evaluate the putative prognostic value using the mutation status of this gene.

\section{Abbreviations}

AA: Anaplastic astrocytoma; ATRX: Alpha-thalassemia/mental retardation syndrome, X-linked; AZT: Azidothymidine; CNS: Central nervous system; GBM: Glioblastoma; IDH: Isocitrate dehydrogenase; MGMTp: O6 methylguanine-DNA methyltransferase promoter; MRI: Magnetic resonance imaging; ODG: Oligodendroglioma; OS: Overall survival; PFS: Progression free survival; TERTp: Telomerase reverse transcriptase promoter; WHO: World Health Organization; WT: Wildtype

\section{Acknowledgements}

This work was supported by funding from Seoul National University Hospital (SNUH0420130350).

\section{Authors' contributions}

YJ Lee and J Koh carried out the molecular genetic studies, participated in the sequence alignment and drafted the manuscript. JK Won performed a statistical analysis. SI Kim, CK Park and SH Choi contributed to the clinical and radiological summary. SH Park has planned, supervised and written this research and all authors read and approved the final manuscript.

\section{Ethics approval and consent to participate}

This study followed the principles of the World Medical Association Declaration of Helsinki and it was approved by the Institutional Review Board of SNUH (IRB No.: 1307-093-505). 


\section{Competing interests}

The authors declare that they have no competing interests.

\section{Publisher's Note}

Springer Nature remains neutral with regard to jurisdictional claims in published maps and institutional affiliations.

\section{Author details}

'Department of Pathology, Seoul National University Hospital, 103 Daehak-ro, Jongno-gu, Seoul 110-799, Republic of Korea. ${ }^{2}$ Department of Pathology, Seoul National University College of Medicine, Seoul, Republic of Korea. ${ }^{3}$ Department of Neurosurgery, Seoul National University, College of Medicine, Seoul, Republic of Korea. ${ }^{4}$ Department of Radiology, Seoul National University, College of Medicine, Seoul, Republic of Korea. ${ }^{5}$ Neuroscience Institute Seoul National University, College of Medicine, Seoul, Republic of Korea.

Received: 6 July 2017 Accepted: 10 August 2017

Published online: 29 August 2017

\section{References}

1. Abudumijiti A, Chan AK, Shi Z, Li Y, Zhang R, Yang R, Li KK, Chung NY, Yao $Y$, Zhou L et al (2017) Adult IDH Wild-type Lower-grade Gliomas Should Be Further Stratified. Neuro-Oncology: Doi 10.1093/neuonc/nox078

2. Andersson U, Osterman P, Sjostrom S, Johansen C, Henriksson R, Brannstrom T, Broholm H, Christensen HC, Ahlbom A, Auvinen A et al (2009) MNS16A minisatellite genotypes in relation to risk of glioma and meningioma and to glioblastoma outcome. Int J Cancer 125:968-972. doi: 10.1002/ijc.24363

3. Arita H, Narita Y, Fukushima S, Tateishi K, Matsushita Y, Yoshida A, Miyakita Y, Ohno M, Collins VP, Kawahara N et al (2013) Upregulating mutations in the TERT promoter commonly occur in adult malignant gliomas and are strongly associated with total 1p19q loss. Acta Neuropathol 126:267-276. doi:10.1007/s00401-013-1141-6

4. Cancer Genome Atlas Research N, Brat DJ, Verhaak RG, Aldape KD, Yung WK, Salama SR, Cooper LA, Rheinbay E, Miller CR, Vitucci M et al (2015) Comprehensive, integrative genomic analysis of diffuse lower-grade Gliomas. N Engl J Med 372:2481-2498. doi:10.1056/NEJMoa1402121

5. Cesare AJ, Reddel RR (2010) Alternative lengthening of telomeres: models, mechanisms and implications. Nat Rev Genet 11:319-330. doi:10.1038/ $\operatorname{nrg} 2763$

6. Chen P, Zou P, Yan Q, Xu H, Zhao P, Gu A (2013) The TERT MNS16A polymorphism contributes to cancer susceptibility: meta-analysis of the current studies. Gene 519:266-270. doi:10.1016/j.gene.2013.02.018

7. Chiba K, Johnson JZ, Vogan JM, Wagner T, Boyle JM, Hockemeyer D (2015) Cancer-associated TERT promoter mutations abrogate telomerase silencing. elife:4. doi:10.7554/eLife.07918

8. Dubbink HJ, Atmodimedjo PN, Kros JM, French PJ, Sanson M, Idbaih A, Wesseling P, Enting R, Spliet W, Tijssen C et al (2016) Molecular classification of anaplastic oligodendroglioma using next-generation sequencing: a report of the prospective randomized EORTC brain tumor group 26951 phase III trial. Neuro-Oncology 18:388-400. doi:10.1093/neuonc/nov182

9. Eckel-Passow JE, Lachance DH, Molinaro AM, Walsh KM, Decker PA, Sicotte H, Pekmezci M, Rice T, Kosel ML, Smirnov IV et al (2015) Glioma groups based on 1p/19q, IDH, and TERT promoter mutations in tumors. N Engl J Med 372:2499-2508. doi:10.1056/NEJMoa1407279

10. Gomez DE, Armando RG, Alonso DF (2012) AZT as a telomerase inhibitor. Front Oncol 2:113. doi:10.3389/fonc.2012.00113

11. Guo C, Pirozzi CJ, Lopez GY, Yan H (2011) Isocitrate dehydrogenase mutations in gliomas: mechanisms, biomarkers and therapeutic target. Curr Opin Neurobiol 24:648-652. doi:10.1097/WCO.0b013e32834cd415

12. Jin TB, Zhang JY, Li G, Du SL, Geng TT, Gao J, Liu QP, Gao GD, Kang LL, Chen $C$ et al (2013) RTEL1 and TERT polymorphisms are associated with astrocytoma risk in the Chinese Han population. Tumour Biol. doi:10.1007/ s13277-013-0947-0

13. Kaloshi G, Benouaich-Amiel A, Diakite F, Taillibert S, Lejeune J, LaigleDonadey F, Renard MA, Iraqi W, Idbaih A, Paris S et al (2007) Temozolomide for low-grade gliomas: predictive impact of $1 \mathrm{p} / 19 \mathrm{q}$ loss on response and outcome. Neurology 68:1831-1836. doi:10.1212/01.wnl.0000262034.26310.a2

14. Killela PJ, Pirozzi CJ, Healy P, Reitman ZJ, Lipp E, Rasheed BA, Yang R, Diplas BH, Wang Z, Greer PK et al (2014) Mutations in IDH1, IDH2, and in the TERT promoter define clinically distinct subgroups of adult malignant gliomas. Oncotarget 5: 1515-1525 Doi 10.18632/oncotarget.1765

15. Killela PJ, Reitman ZJ, Jiao Y, Bettegowda C, Agrawal N, Diaz LA Jr, Friedman AH, Friedman H, Gallia GL, Giovanella BC et al (2013) TERT promoter mutations occur frequently in gliomas and a subset of tumors derived from cells with low rates of self-renewal. Proc Natl Acad Sci U S A 110:6021-6026. doi:10.1073/pnas.1303607110

16. Labussiere M, Di Stefano AL, Gleize V, Boisselier B, Giry M, Mangesius S, Bruno A, Paterra R, Marie Y, Rahimian A et al (2014) TERT promoter mutations in gliomas, genetic associations and clinico-pathological correlations. Br J Cancer 111:2024-2032. doi:10.1038/bjc.2014.538

17. Li G, Jin TB, Wei XB, He SM, Liang HJ, Yang HX, Cui Y, Chen C, Cai LB, Gao GD (2012) Selected polymorphisms of GSTP1 and TERT were associated with glioma risk in Han Chinese. Cancer Epidemiol 36:525-527. doi:10.1016/ j.canep.2012.06.008

18. Liu X, Wu G, Shan Y, Hartmann C, von Deimling A, Xing M (2013) Highly prevalent TERT promoter mutations in bladder cancer and glioblastoma. Cell Cycle 12:1637-1638. doi:10.4161/cc.24662

19. Louis DN, Perry A, Reifenberger G, von Deimling A, Figarella-Branger D, Cavenee WK, Ohgaki H, Wiestler OD, Kleihues P, Ellison DW (2016) The 2016 World Health Organization classification of tumors of the central nervous system: a summary. Acta Neuropathol 131:803-820. doi:10.1007/s00401-016-1545-1

20. Makar AB, McMartin KE, Palese M, Tephly TR (1975) Formate assay in body fluids: application in methanol poisoning. Biochem Med 13:117-126

21. Mosrati MA, Malmstrom A, Lysiak M, Krysztofiak A, Hallbeck M, Milos P, Hallbeck AL, Bratthall C, Strandeus M, Stenmark-Askmalm M et al (2015) TERT promoter mutations and polymorphisms as prognostic factors in primary glioblastoma. Oncotarget 6:16663-16673. doi:10.18632/oncotarget.4389

22. Nonoguchi N, Ohta T, Oh JE, Kim YH, Kleihues P, Ohgaki H (2013) TERT promoter mutations in primary and secondary glioblastomas. Acta Neuropathol 126:931-937. doi:10.1007/s00401-013-1163-0

23. Pekmezci M, Rice T, Molinaro AM, Walsh KM, Decker PA, Hansen H, Sicotte H, Kollmeyer TM, McCoy LS, Sarkar G et al (2017) Adult infiltrating gliomas with WHO 2016 integrated diagnosis: additional prognostic roles of ATRX and TERT. Acta Neuropathol 133:1001-1016. doi:10.1007/s00401-017-1690-1

24. Ruden M, Puri N (2013) Novel anticancer therapeutics targeting telomerase. Cancer Treat Rev 39:444-456. doi:10.1016/j.ctrv.2012.06.007

25. van den Bent MJ, Brandes AA, Taphoorn MJ, Kros JM, Kouwenhoven MC, Delattre JY, Bernsen HJ, Frenay M, Tijssen CC, Grisold W et al (2013) Adjuvant procarbazine, lomustine, and vincristine chemotherapy in newly diagnosed anaplastic oligodendroglioma: long-term follow-up of EORTC brain tumor group study 26951. J Clin Oncol 31:344-350. doi:10.1200/JCO. 2012.43.2229

26. Verhaak RG, Hoadley KA, Purdom E, Wang V, Qi Y, Wilkerson MD, Miller CR, Ding L, Golub T, Mesirov JP et al (2010) Integrated genomic analysis identifies clinically relevant subtypes of glioblastoma characterized by abnormalities in PDGFRA, IDH1, EGFR, and NF1. Cancer Cell 17:98-110. doi: 10.1016/j.ccr.2009.12.020

27. Zhu J, Zuo J, Xu Q, Wang X, Wang Z, Zhou D (2011) Isocitrate dehydrogenase mutations may be a protective mechanism in glioma patients. Med Hypotheses 76:602-603. doi:10.1016/j.mehy.2011.01.011

\section{Submit your next manuscript to BioMed Central and we will help you at every step:}

- We accept pre-submission inquiries

- Our selector tool helps you to find the most relevant journal

- We provide round the clock customer support

- Convenient online submission

- Thorough peer review

- Inclusion in PubMed and all major indexing services

- Maximum visibility for your research

Submit your manuscript at www.biomedcentral.com/submit 